\title{
BiGlobal stability analysis of steady flow in constricted channel geometries
}

\author{
R. E. Pitt ${ }^{1, *, \dagger}$, S. J. Sherwin ${ }^{1}$ and V. Theofilis ${ }^{2}$ \\ ${ }^{1}$ Aeronautics Department, Imperial College, London, SW7 2AZ, U.K. \\ ${ }^{2}$ Universidad Politécnica de Madrid, Escuela Tecnica Superior Ingenieros Aeronáuticos, Departamento de \\ Motopropulsión y Termofluidodinámica, Pza. Cardenal Cisneros, 3, E-28040 Madrid, Spain
}

\begin{abstract}
SUMMARY
In this paper we apply a BiGlobal stability analysis technique to measure the stability of two-dimensional constricted channel flows to three-dimensional perturbations. Critical Reynolds numbers and spanwise perturbation wavelengths are presented for three instabilities of steady flow in constricted channels.
\end{abstract}

KEY WORDS: BiGlobal; stability; constricted; channel

\section{INTRODUCTION}

The flow through a constricted channel is an interesting fluid mechanical problem that has seen renewed interest in the past few decades due to attempts to model the flow in simplified arterial geometries. The numerical solution of the flows in these geometries is not easy, however. The high local velocities at the constriction and the need for a fine discretisation results in a greatly reduced time-step when considering the CFL stability restriction associated with an explicit treatment of the advection operator. The resulting high computational time can make a thorough investigation of the many parameters involved such as geometry, inflow waveform and Reynolds number, prohibitively expensive.

A BiGlobal stability analysis [1] can alternatively be employed to study the laminar instabilities and transitions occurring within the constriction. Unlike classical stability analysis where a one-dimensional base flow is considered and the other two spatial directions are harmonically expanded, in the BiGlobal stability analysis both the basic state and the amplitude functions of small-amplitude disturbances superimposed upon the basic state are non-periodic two-dimensional functions; the third spatial direction is considered homogeneous and expanded

*Correspondence to: R. E. Pitt, Aeronautics Department, Imperial College, London, SW7 2AZ, U.K.

$\dagger$ E-mail: robin.pitt@imperial.ac.uk 


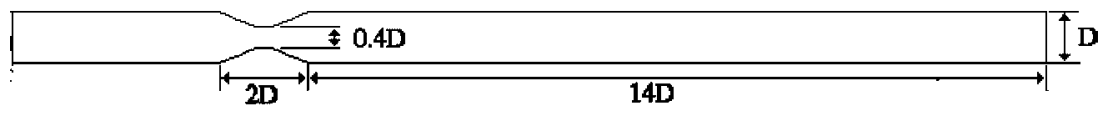

Figure 1. Geometry of $60 \%$ occluded channel stenosis model.

harmonically in Fourier wave numbers $\beta$. The method is thus suited to investigation of the stability of flows with homogeneity of geometry in one dimension, for example channel, cylinder or axisymmetric geometries.

It should be noted that in some cases (e.g. the flow in a straight pipe) a linearized eigenvalue analysis such as this would predict the flow to be unconditionally stable at all Re. Under these circumstances the sensitivity of the flow to non-modal finite perturbations can be evaluated by using a psuedo-spectral technique [2].

In this paper, the geometry we consider is a plane channel geometry, infinite in the $z$ direction, with a prescribed contraction and subsequent re-expansion in the $y$-direction, shown with length scales marked in Figure 1. We consider a steady inflow. Below a Reynolds number of approximately 125 , flow in the symmetric $60 \%$ constricted channel has a unique solution; it is symmetric and two-dimensional. Above this critical value of $R e$ a primary instability occurs: a pitchfork bifurcation resulting in one of two stable asymmetric flows [3]. This instability, biasing the shear layers shed from the constriction throat to one side, can be attributed to the Coanda effect. If the Re of the two-dimensional asymmetric base flow is then further increased then a second critical Reynolds number is reached; a secondary instability occurs that results in three-dimensionality of the flow.

In this paper we first present a brief introduction to the numerical method involved in this BiGlobal stability analysis. We then demonstrate how the stability of these primary and secondary base flows is affected by $R e$ and $\beta$. Finally we show how the onset of threedimensionality is affected by the introduction of asymmetry into the channel geometry. These results are determined in terms of the value of the dominant eigenvalue (indicating the instability modal growth rate) as curves of neutral stability in the parameter space $(\beta, R e)$.

\section{NUMERICAL METHODOLOGY}

We take as the governing equations for arterial flow the incompressible Newtonian NavierStokes equations

$$
\frac{\partial \mathbf{u}}{\partial t}=-\mathbf{N}(\mathbf{u})-\nabla p+\frac{1}{R e} \nabla^{2} \mathbf{u} \quad \text { in } \Omega
$$

together with the continuity requirement

$$
\nabla \cdot \mathbf{u}=0 \text { in } \Omega
$$

where $\mathbf{u}$ is the three-dimensional velocity field, $p$ is the fluid pressure, and $R e$ is the Reynolds number $R e=U D / v$. For our purposes the length scale $D$ taken in the definition of the Reynolds number is the channel height (see Figure 1), and the velocity scale $U$ is the temporally and spatially averaged inflow velocity $(\tilde{U})$. $\mathbf{N}(\mathbf{u})$ is the non-linear advection operator $\mathbf{N}(\mathbf{u})=$ 
$(\mathbf{u} \cdot \nabla) \mathbf{u}$. Equation (1) is subject to no-slip boundary conditions at the walls, a prescribed plane Poiseuille velocity distribution at the inflow, and conditions of zero pressure and zero outward normal derivatives of velocity at the outflow.

We decompose the instantaneous flow field into a two-dimensional base flow, $\mathbf{U}$, and a small perturbation $\mathbf{u}^{\prime}$ :

$$
\mathbf{u}(x, y, z, t)=\mathbf{U}(x, y, t)+\mathbf{u}^{\prime}(x, y, z, t)
$$

$\mathbf{U}$ is a solution of Equation ( 1 ) on a two-dimensional computational domain, $\Omega$, which is invariant in the $z$-direction (the direction of homogeneity). For the work of this paper the base flow is steady $(\partial \mathbf{U} / \partial t=0)$, although it may also be time periodic, in which case a Floquet stability analysis is applied.

Placing the definition (3) into (1) and neglecting as small the terms corresponding to the product of the small perturbations we arrive at the linearized Navier-Stokes equations:

$$
\frac{\partial \mathbf{u}^{\prime}}{\partial t}=-\mathbf{D N}\left(\mathbf{u}^{\prime}\right)-\nabla p^{\prime}+\frac{1}{R e} \nabla^{2} \mathbf{u}^{\prime} \quad \text { in } \Omega
$$

where $\mathbf{u}^{\prime}$ is again constrained to be divergence free (satisfying (2)), a situation which is maintained by the perturbation pressure field $p^{\prime}$. In order to ensure that the disturbance flow satisfies the same boundary conditions as the complete flow, $\mathbf{u}^{\prime}$ is constrained to be zero at the Dirichlet boundaries and shares the same outflow condition as imposed previously on $\mathbf{u}$. DN is the linearized advection operator:

$$
\mathbf{D N}=\left(\mathbf{u}^{\prime} \cdot \nabla\right) \mathbf{U}+(\mathbf{U} \cdot \nabla) \mathbf{u}^{\prime}
$$

For the case of steady base flow $\mathbf{D N}$ is constant. Equation (4) can be written more compactly as

$$
\frac{\partial \mathbf{u}^{\prime}}{\partial t}=\mathbf{L}\left(\mathbf{u}^{\prime}\right)
$$

where the linear operator $\mathbf{L}\left(\mathbf{u}^{\prime}\right)$ represents the right hand side of (4).

For steady base flows, solutions of (4) comprise a sum of exponential functions of the form $\tilde{\mathbf{u}}(x, y, z, t) \mathrm{e}^{\sigma t}$. We consider the exponents $\sigma$. A mode is linearly unstable (will grow in time) if the real part of this exponent is greater than zero.

A simplification to the form of $\mathbf{u}^{\prime}$ can be made due to the homogeneity of the domain and the assumption that it is infinite in the $z$-direction, by expressing the general perturbation as the Fourier integral [4]:

$$
\mathbf{u}^{\prime}(x, y, z, t)=\int_{-\infty}^{\infty} \hat{\mathbf{u}}(x, y, \beta, t) \mathrm{e}^{\mathrm{i} \beta z} \mathrm{~d} \beta
$$

This also has the effect of modifying the gradient operator wherever it is used so that $\nabla \equiv(\partial / \partial x, \partial / \partial y,-\mathrm{i} \beta)$. The linearity of (4) ensures that perturbation modes with different spanwise wave number $\beta$ do not couple, and thus can be calculated separately.

In order to find the dominant (most unstable) exponents we define an operator $\mathbf{A}$ describing the evolution of $\mathbf{u}^{\prime}$ over an arbitrary period $T$, chosen for computational convenience:

$$
\mathbf{u}_{n+1}^{\prime}=\mathbf{A}\left(\mathbf{u}_{n}^{\prime}\right)
$$


where $\mathbf{u}_{n}^{\prime}$ is the perturbation field after $n$ time-stepping periods of time $T$. The action of $\mathbf{A}$ is the time integrated effect of the operator $\mathbf{L}$ on an initially infinitesimal perturbation over time $T$ :

$$
\mathbf{A}\left(\mathbf{u}^{\prime}\right)=\exp \left(\int_{0}^{T} \mathbf{L}\left(\mathbf{u}^{\prime}\right) \mathrm{d} t\right)
$$

The eigenmodes of A correspond to the instability eigenmodes of the system, $\tilde{\mathbf{u}}$. The exponents, dictating the linear stability of the base flow in question and corresponding to the growth rates of the eigenmodes, are calculated via the relation $\sigma=(\ln (\mu) / T)$, where $\mu$ is the eigenvalue of $\mathbf{A}$. Instability occurs when the value of the dominant exponent becomes greater than zero.

The action of $\mathbf{A}$ is approximated by integrating (4) over $T / \Delta t$ time-steps. This is performed by modification of an existing spectral $/ h p$ element solver for solution of the Navier-Stokes equations (1) on three-dimensional domains with $z$-direction homogeneity. The non-linear advection operator $\mathbf{N}$ must be replaced with its linearized counterpart $\mathbf{D N}$, and the gradient operator must be modified as previously stated.

All of the base flows were calculated using an unsteady two-dimensional spectral/hp NavierStokes solver, on the same meshes as used for the subsequent stability analysis.

The eigenvalues of $\mathbf{A}$ are found via the time integration of (4), using the Arnoldi method, avoiding the high memory requirements of a direct method. For the work in this paper a low Krylov subspace dimension was used, but sufficiently large for converged eigenvalues to be obtained. The spectral $/ h p$ method of spatial discretisation, chosen for this work for its favourable convergence properties, is described in the context of stability analysis by Theofilis et al. [5]. The calculations in this paper have been performed on stenoses of $60 \%$ occlusion, using symmetric and asymmetric meshes of approximately 1600 elements. All calculations were performed using a maximum expansion base polynomial order of 6 , giving approximately 78000 local degrees of freedom per variable.

\section{RESULTS AND DISCUSSION}

\subsection{Base flow characteristics}

Figure 2(a) shows the vorticity contours of the steady symmetric base flow at approximately the point of bifurcation, $R e=125$. This flow was generated using a symmetric boundary condition along the channel centre-line and as such may be unstable to two-dimensional perturbations. The flow is unable to expand rapidly enough to follow the curve of the wall as it leaves the constriction, and instead separates symmetrically. From the vorticity plot of Figure 2(a) we observe the growth of two boundary layer regions in the contraction, which separate into two shear layers trailing downstream of the expansion. The separation points are clearly visible in Figure 2(b). These separated shear layers provide the boundaries between the jet-type flow emanating from the constriction, and two recirculation regions driven by the jet.

Figure 2(b) shows streamlines of the symmetric flow. The recirculation regions on either side of the jet are clearly visible, attached to the top and bottom surfaces of the channel, immediately downstream of the constriction. As the jet progresses away from the stenosis, 


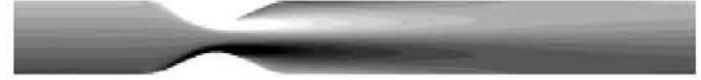

(a)

(c)

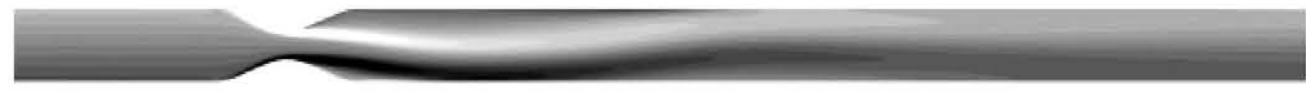

(d)

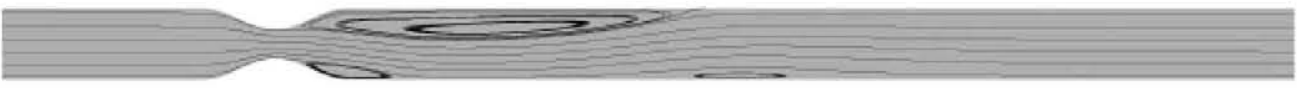

(e)

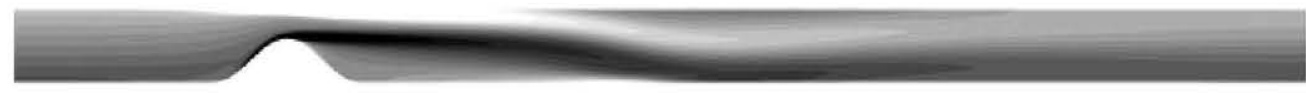

(f)

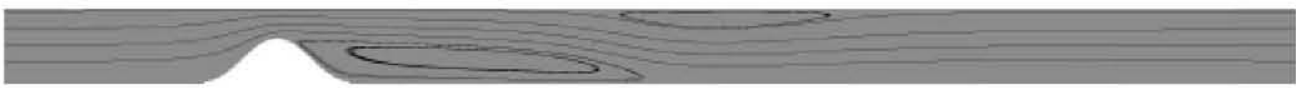

Figure 2. Base flows: (a) vorticity contours and (b) streamlines of symmetrically constrained flow in symmetric geometry at $R e=125$; (c) and (d) unconstrained flow in symmetric geometry at $R e=300$; and (e) and (f) flow in asymmetric geometry at $R e=250$.

it expands to reattach to the upper and lower channel walls, and the axial velocity profile asymptotes to that of fully developed Poiseuille flow under the effect of viscous momentum diffusion. After the flow has travelled only 11 channel diameters from the constriction throat the peak flow velocity across the channel has returned to the fully developed non-dimensional value of 1.5 (to three significant figures), and measurements taken at stations further downstream show no significant deviation from this profile.

Figure 2(c) shows the vorticity field in the unconstrained base flow in the symmetric geometry, near the onset of the secondary instability, at a Reynolds number of 300 . In order to investigate the onset of three-dimensionality the flow is constrained to be two-dimensional, implicit in the formulation of the two-dimensional flow solver. It is transparent that the flow field is extremely asymmetric, as the jet veers steeply downward upon exiting the constriction. The streamlines (Figure 2(d)) show the same deviation of the bulk momentum, and also show the imbalance of the sizes of the two recirculation regions. The greatest downward deflection of the jet occurs at 3D from the constriction, and it is then deflected upward to a maximum $y$-position at approximately 7D before slowly settling back to the central position of the Poiseuille flow at approximately 27D from the throat.

Figures 2(e) and (f) show the base flow in the asymmetric $60 \%$ constricted geometry at $R e=250$. The base flow is much like the asymmetric flow in the symmetric geometry, although the jet adheres to the top surface immediately after the constriction. There is therefore no recirculation cell on the upper surface at this point.

\subsection{Linearised stability analysis}

Figure 3(a) shows the neutral linearized stability curve for the primary instability. It can be seen that symmetry breaking within the plane will occur at a critical Re just below 125 . The curve of Figure 3(a) implies that this occurs with an instability wavelength that is nonzero, introducing a mild three-dimensionality to the flow. However, the shape of the curve 

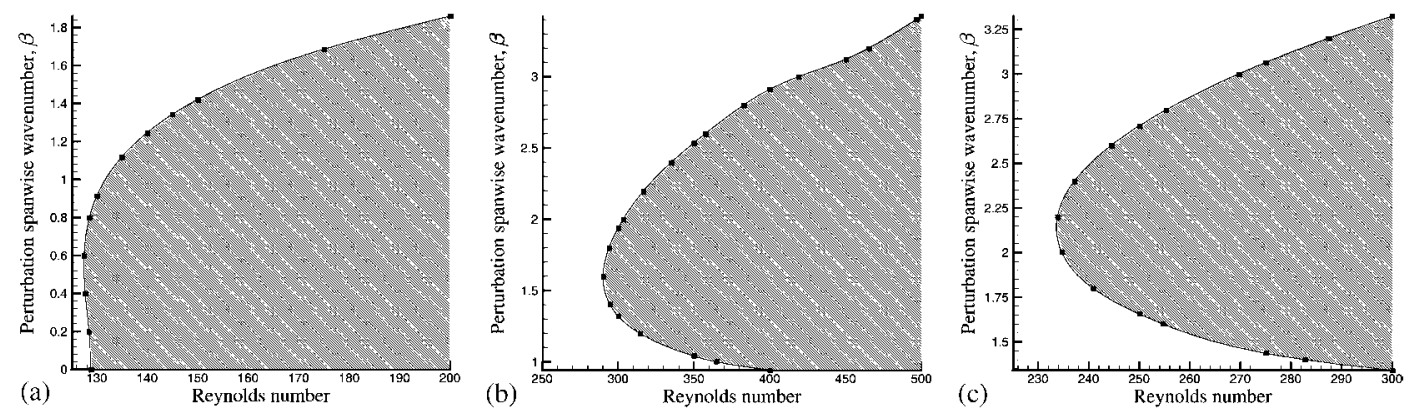

Figure 3. Neutral stability curves: (a) symmetrically constrained flow in symmetric geometry; (b) unconstrained flow in symmetric geometry following primary instability; and (c) flow in asymmetric geometry. Shaded regions are linearly unstable.

indicates that the flow becomes rapidly linearly unstable to a wide range of $\beta$, including two-dimensional perturbations, and it should be noted that stable two-dimensional flows exist beyond this $R e$. This value of the critical $R e$ is in accord with experimental studies [6], and two-dimensional simulations with no in-plane constraint.

Figure 3 (b) shows the neutral stability curve for the dominant mode leading to the onset of three-dimensionality. In this case the critical $R e$ is approximately 290 , occurring with a spanwise wave number of 1.6 , or approximately $4 \mathrm{D}$.

Figure $3(\mathrm{c})$ shows the curve for the onset of three-dimensionality in the asymmetric geometry. The critical $R e$ is approximately 235 in this case. Thus for steady flow the effect of geometrical asymmetry is clearly destabilising. Also, the instability occurs at a higher wave number $(\approx 2.2)$, corresponding to a shorter instability wavelength of $2.9 \mathrm{D}$, although the degree of constriction is the same in both cases.

\section{CONCLUSIONS}

We have successfully isolated the critical Reynolds numbers and spanwise wave numbers for three linear instabilities of steady constricted channel flows, and shown the effect of constriction asymmetry to be destabilising. In addition, the calculations furnish us with full details of the instability modes arising.

\section{REFERENCES}

1. Theofilis V. Advances in global linear instability and control. Progress in Aeronautical Sciences 2003; 39(4): 249-315.

2. Trefethen LN, Trefethen AE, Reddy SC, Driscoll TA. Hydrodynamic stability without eigenvalues. Science 1993; 261:578-584.

3. Sobey IJ, Drazin PG. Bifurcations of two-dimensional channel flows. Journal of Fluid Mechanics 1986; 171: 263-287.

4. Barkley D, Henderson RD. Three-dimensional Floquet stability analysis of the wake of a circular cylinder. Journal of Fluid Mechanics 1996; 322:215-241. 
5. Theofilis V, Barkley D, Sherwin SJ. Spectral/hp element technology for flow instability and control. Aeronautical Journal 2002; 106:615-619.

6. Cherdron W, Durst F, Whitelaw JH. Asymmetric flows and instabilities in symmetric ducts with sudden expansions. Journal of Fluid Mechanics 1976; 84(1):13-31.

7. Caro CG, Fitzgerald JM, Schroter RC. Atheroma and arterial wall shear observation, correlation and proposal of a shear dependent mass transfer mechanism for atherogenesis. Proceedings of the Royal Society, Series $B$ $1971 ; 177: 109-159$. 